\title{
Preliminary Results of Relationship between Preoperative Walking Ability and Magnetic Resonance Imaging Morphology in Patients with Lumbar Canal Stenosis: Comparison between Trefoil and Triangle Types of Spinal Stenosis
}

\author{
Parisa Azimi ${ }^{1}$, Taravat Yazdanian ${ }^{2}$, Edward C. Benzel $^{3}$ \\ ${ }^{1}$ Functional Neurosurgery Research Center, Shahid Beheshti University of Medical Sciences, Tehran, Iran \\ ${ }^{2}$ School of Medicine, Capital Medical University, Beijing, China \\ ${ }^{3}$ Department of Neurosurgery, Cleveland Clinic Foundation, Cleveland, OH, USA
}

\section{Study Design: Cross-sectional.}

Purpose: To examine the relationship between magnetic resonance imaging (MRI) morphology stenosis grades and preoperative walking ability in patients with lumbar canal stenosis (LCS).

Overview of Literature: No previous study has analyzed the correlation between MRI morphology stenosis grades and walking ability in patients with LCS.

Methods: This prospective study included 98 consecutive patients with LCS who were candidates for surgery. Using features identified in T2-weighted axial magnetic, stenosis type was determined at the maximal stenosis level, and only trefoil and triangle stenosis grade types were considered because of sufficient sample size. Intraobserver and interobserver reliability were assessed by calculating weighted kappa coefficients. Symptom severity was evaluated via the Japanese Orthopedic Association Back Pain Evaluation Questionnaire (JOABPEQ). Walking ability was assessed using the Self-Paced Walking Test (SPWT) and JOABPEQ subscales. Demographic characteristics, SPWT scores, and JOABPEO scores were compared between patients with trefoil and triangle stenosis types. Results: The mean patient age was 58.1 (standard deviation, 8.4) years. The kappa values of the MRI morphology stenosis grade types showed a perfect agreement between the stenosis grade types. The trefoil group $(n=53)$ and triangle group $(n=45)$ showed similar preoperative JOABPEQ subscale scores (e.g., low back pain, lumbar function, and mental health) and were not significantly different in age, BMI, duration of symptoms, or lumbar stenosis levels (all $p>0.05$ ); however, trefoil stenosis grade type was associated with a decreased walking ability according to the SPWT and JOABPEO subscale scores.

Conclusions: These findings suggest preoperative walking ability is more profoundly affected in patients with trefoil type stenosis than in those with triangle type stenosis.

Keywords: Japanese Orthopedic Association Back Pain Evaluation Questionnaire; Comparison; Lumbar canal stenosis; MRI morphology stenosis grades

\footnotetext{
Received Dec 10, 2016; Revised Jan 16, 2017; Accepted Jan 31, 2017

Corresponding author: Parisa Azimi

Functional Neurosurgery Research Center, Shohada Tajrish Hospital, Shahid Beheshti University of Medical Sciences,

Sharadari St., Tajrish Square, Tehran 1989934148, Iran

Tel: 98-2122749204, Fax: +98-2188265188, E-mail: parisa.azimi@gmail.com
} 


\section{Introduction}

Degenerative changes in the vertebrae (bones), discs, muscles, and ligaments (connective tissues) are the most common cause of lumbar spinal stenosis (LCS), and LCS prevalence increases with age [1]. Symptoms of LCS typically include leg pain, low back pain, numbness, tingling, and/or burning [2]. Symptoms often worsen with prolonged walking. The typical walking distance in severe cases is only $5-10 \mathrm{~m}$, and the symptoms are usually relieved upon anterior flexion of the spine [3].

Walking ability is considered essential for most activities of daily living [4], and of its assessment is a standard part of evaluating patients with LCS [5]. Routine magnetic resonance imaging (MRI) is the standard method used to clinically assess patients with lumbar spinal stenosis (LSS) [6]. On the other hand, using MRI, several grading systems, such as the classification system described by Menon et al. [7], have been developed for the diagnostic criteria of patients with LCS [7-11]. To date, several studies have analyzed the relationship between MRI morphology stenosis grade and symptom severity in patients with LCS; however, the use of MRI morphology grade remains controversial [7-11]. Walking ability can be assessed by both self-reported measures such as Japanese Orthopedic Association Back Pain Evaluation Questionnaire (JOABPEQ) [12] and objective evaluations such as the Self-Paced Walking Test (SPWT) [13]. To our knowledge, only one previous study showed a positive correlation between the dural sac cross-sectional area (DCSA) obtained by conventional MRI and walking ability in patients with LCS [10]. In addition, there are no studies of the correlation between MRI morphology stenosis grade and the walking ability according to JOABPEQ and SPWT scores in patients with LCS. Therefore, for decision-making purposes, it remains unknown whether stenosis type affects walking ability in patients with LCS. The purpose of the present study was to assess the effect of MRI morphology stenosis grade on preoperative walking ability in patients with LCS.

\section{Materials and Methods}

\section{Patients and data collection}

In this prospective study, conducted between February 2014 and January 2016, a total of 98 consecutive patients who were surgical candidates for LCS were recruited from two teaching hospitals in Tehran, Iran. Although all recruited patients had central canal stenosis, some patients also had LCS associated with a lateral recess. All patients had the typical symptoms associated with LCS, including neurogenic intermittent claudication and leg pain, radicular or combined type, and/or numbness. LCS diagnosis was established using both clinical and radiological methods, and the initial diagnosis was confirmed by at least one other surgeon. All patients received conservative treatment for a minimum of 6 months [14]. Using patterns on T2 axial MRI, stenosis type was determined for each patient. The type of axial imaging features in patients with LCS were categorized as symmetrical or asymmetrical, with five subtypes. The morphological types were labeled as" trefoil", "triangle", "cat's eye," "pin-hole," and "nohole" varieties [7]. The trefoil type has three subtypes (A, B, and $\mathrm{C}$ ), whereas the triangle type has two subtypes (large and small). The large triangle is an isosceles triangle elongated in the antero-posterior (AP) direction and the disk is largely noncontributing to the canal compromise. In the small triangle, the shape is more equilateral and the AP dimensions are also significantly smaller [7]. The asymmetrical trefoil and triangle patterns were defined by Menon et al. [7]. Examples of symmetrical "trefoil" and "triangle" patterns are shown in Fig. 1. Detailed LCS patterns have been described by Menon et al. [7]. MRI morphology was measured at the maximal stenosis level. Demographics, including age, body weight $(\mathrm{kg})$, duration of symptoms (months), JOABPEQ score, SPWT (m), stenosis level, and types of LCS were assessed. There were no limitations for patient selection regarding the types of LCS, age, or other characteristics. We excluded all patients with a history of prior lumbar spine surgery and spinal anomalies from the study. In addition, patients with hip and knee disease (including a history of hip fracture), which may severely affect their walking ability were excluded from the study. Cauda equina cases were also excluded.

\section{Measures}

\section{1) JOABPEQ}

The JOABPEQ score for assessing low back pain was calculated. It is a disease-specific tool and contains 25 items divided into five subscales: (1) low back pain (four items); (2) mental health (seven items); (3) lumbar function (six items); (4) walking ability (five items); and (5) social function (four items). The score for each subscale ranged from 


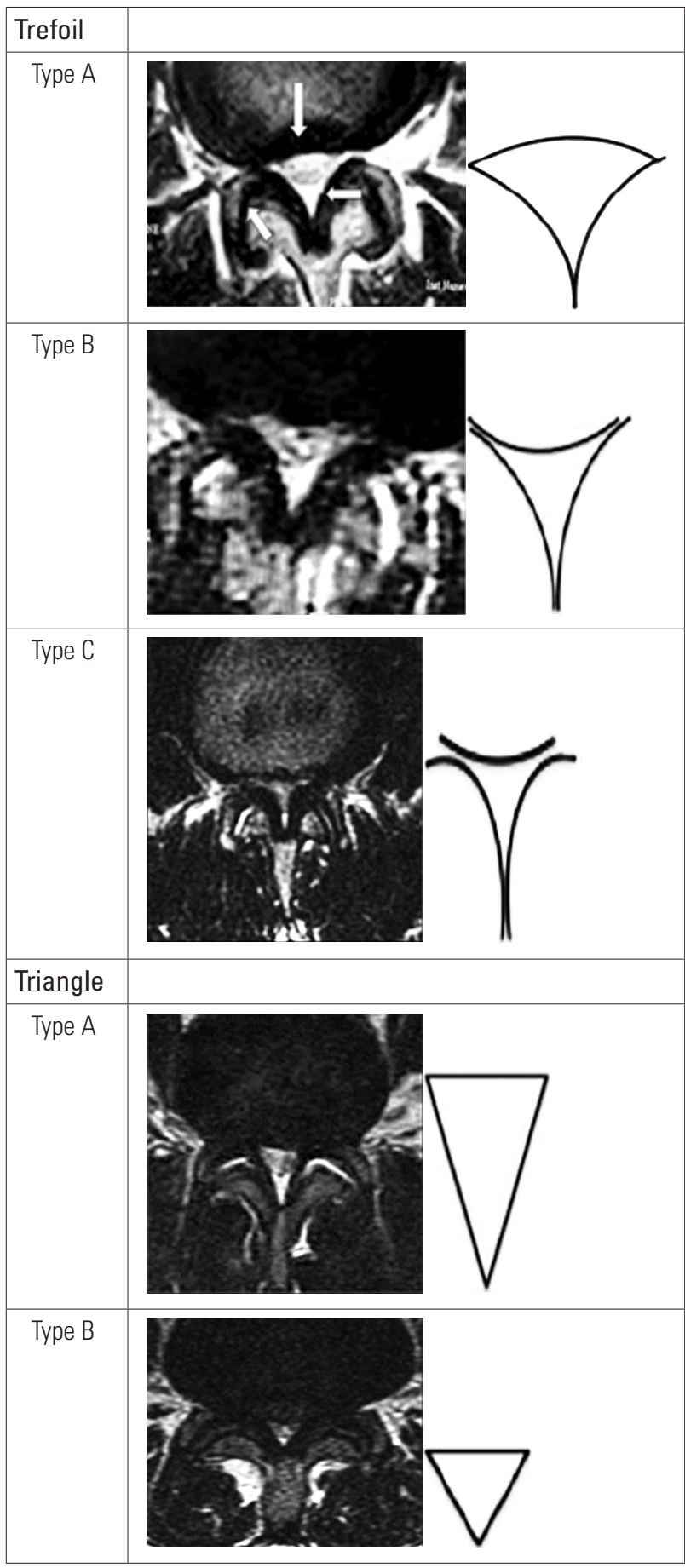

Fig. 1. Examples of a symmetrical "trefoil" and "triangle" configuration of spinal canal morphology in patients with lumbar canal stenosis as derived from reference (reprinted from Menon et al. J Spinal Disord Tech 2015;28:12-8, with permission of Wolters Kluwer Health, Inc. [7]).

0 to 100 and decreases with worsening disability [12].

\section{2) SPWT}

SPWT is a measure for walking ability, defined as the distance a person with LSS can walk without support on a level surface at a self-selected speed until he or she must rest due to symptoms of back or leg pain $[13,15,16]$.

\section{3) DSCA}

The dural sac cross-sectional area (DSCA) was measured in $\mathrm{mm}^{2}$ at the maximal stenosis level, either via CT or axial MRI sequences obtained at the disc level using the Hamanishi technique $[17,18]$.

\section{Statistical analysis}

Descriptive statistics (e.g., means, standard deviations, and percentages) were used to explore the quantitative and categorical study variables. A Student $t$-test was used for continuous data and the $\chi^{2}$ test was used for categorical data. The statistical significance threshold was defined as $p<0.05$. The SPSS ver. 18 software package (SPSS Inc., Chicago, IL, USA) was used for all analyses.

\section{Intra- and interobserver reliability assessment of types of MRI morphology stenosis grades}

Two independent observers classified each case twice within a 1-week interval to measure intraobserver and interobserver reliability. The weighted kappa coefficient was calculated for each spine surgeon on the basis of their first and second data report and comparison of the kappa coefficient between spine surgeons. The kappa value varied between 0 and 1; a greater kappa value indicated a higher concordance rate. A kappa values of $0-0.20$ indicated a slight agreement, 0.21-0.40 indicated a fair agreement, $0.41-0.60$ indicated a moderate agreement, $0.61-0.80$ indicated a substantial perfect agreement, and $\geq 0.81$ indicated an almost perfect agreement according to the interpretation by Landis and Koch [19].

\section{Ethics}

The Ethics Committee of Shahid Beheshti University of Medical Sciences, Tehran, Iran, approved the study and agreed with the consent procedure.

\section{Results}

Of the 121 patients, 98 patients with trefoil and triangle stenosis were included in this study. To prevent bias be- 
cause of small sample sizes, the remaining 23 patients in the "cat's eye," group $(n=6)$, "indefinable shapes" group $(n=5)$, and the "pin-hole" group $(n=12)$ and were excluded from the analysis. The mean age of the patients was 58.1 (standard deviation, 8.4) years (range, 35-83 years). The clinica characteristics as well as MRI morphology, JOABPEQ scores, DSCA, and SPWT results of the patients are shown in Table 1. The majority of patients (81.0\%) had trefoil and triangle types of stenosis. The age, level of lumbar stenosis, or symptom duration between the trefoil group ( $\mathrm{n}=53$; symmetrical, $\mathrm{n}=36)$ and triangle group ( $\mathrm{n}=45$; symmetrical, $\mathrm{n}=33$ ) were not significantly different ( $p>0.4$ for all).

The kappa values for the types of MRI morphology stenosis grades for the intraobserver reliability was 0.84 (range, 0.80-0.94) and for the interobserver reliability was 0.82 (range, 0.81-0.92), indicating a substantial per- fect agreement. Trefoil stenosis, was associated with a worse walking ability and a decreased social life function compared to triangle stenosis (JOABPEQ, 14.7 vs. 42.3; $p<0.001$ ); however, no significant differences existed between the two groups regarding low back pain, lumbar function, mental health, and DCSA were found. In addition, significant differences between trefoil and triangle stenosis were found for walking ability according to SPWT scores (SPWT, 231.7 vs. 351.6; $p=0.034$ ) (Table 1).

\section{Discussion}

The main finding of our study was that there was a significant difference in the walking ability between the trefoil and triangle types of MRI morphology. Therefore, MRI morphology appears to be a simple and useful tool for assessing the walking ability in patients with LCS; however,

Table 1. Demographic data and health status tools according to types of MRI morphology stenotic grades in patients with lumbar spinal canal stenosis $(\mathrm{n}=98)$

\begin{tabular}{|c|c|c|c|}
\hline \multirow{2}{*}{ Characteristics } & \multicolumn{2}{|c|}{ Patterns of lumbar canal stenosis ${ }^{a)}$} & \multirow{2}{*}{$p$-value } \\
\hline & Trefoil $(n=53)$ & Triangle $(n=45)$ & \\
\hline Age (yr) & $57.3(7.7)$ & $56.4(9.2)$ & 0.765 \\
\hline Range & $35-82$ & $41-83$ & \\
\hline Sex (male, \%) & 52.8 & 51.1 & 0.865 \\
\hline Body weight (kg) & $83.3(9.1)$ & $84.1(9.3)$ & 0.754 \\
\hline \multicolumn{4}{|l|}{ Lumbar stenosis levels } \\
\hline One-level & $32(60.4)$ & $27(60.0)$ & 0.947 \\
\hline Two-level & $19(35.8)$ & $17(37.8)$ & 0.564 \\
\hline Three-level & $2(3.8)$ & $1(2.3)$ & 0.492 \\
\hline \multicolumn{4}{|l|}{ Symptoms } \\
\hline Duration of symptoms (mo) & $42.7(18.4)$ & $38.7(17.6)$ & 0.553 \\
\hline $\mathrm{SPWT}(\mathrm{m})$ & $231.7(221.1)$ & $351.6(271.3)$ & 0.034 \\
\hline DSCA $\left(\mathrm{mm}^{2}\right)^{\mathrm{b})}$ & $76(20.2)$ & $87(16.3)$ & 0.06 \\
\hline Range & $13-123$ & $25-145$ & \\
\hline \multicolumn{4}{|l|}{ JOABPEO } \\
\hline Low back pain & $25.1(29.0)$ & $29.6(13.7)$ & 0.33 \\
\hline Lumbar function & $34.8(28.1)$ & $27.5(32.6)$ & 0.537 \\
\hline Walking ability & $14.7(17.2)$ & $42.3(28.5)$ & $<0.001$ \\
\hline Social life function & $32.7(18.9)$ & $47.0(16.6)$ & 0.006 \\
\hline Mental health & $43.4(12.4)$ & $51.3(16.3)$ & 0.06 \\
\hline
\end{tabular}

Values are presented as number (\%).

MRI, magnetic resonance imaging; SPWT, Self-Paced Walking Test; DCSA, the dural sac cross-sectional area; JOABPEQ, Japanese Orthopedic Association Back Pain Evaluation Questionnaire.

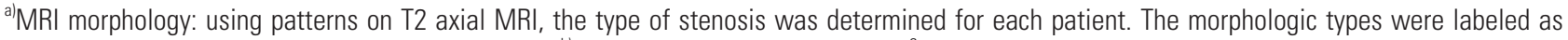

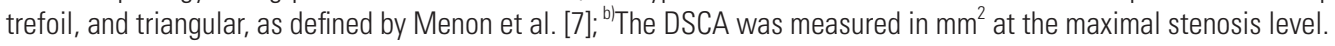


there may be other underlying pathobiological mechanisms to be revealed in association with this disease [20].

Although, evidence from prospective studies measuring preoperative and postoperative walking ability in patients with LSS suggests that these patients significantly improved after surgery [21-24], the relationship between the MRI morphology stenosis grade and preoperative walking ability in patients with LCS is currently unknown. To date no investigations have shown an association between clinical symptoms and imaging findings [1,6,9]; however, significant differences between patients with the two stenosis grade types according to walking ability, social life, and SPWT scores were found in the present study. One previous study reported that a linear association existed between the minimum cross-sectional area (mCSA) of the cauda equina and the patients' walking ability, indicating that a smaller mCSA was associated with a shorter preoperative walking ability [10]. Overall, DCSA in the trefoil type is less than that in the triangle type; however, the difference was not statistically significant (Table 1), which may be the source of the findings in the present study. Major narrowing of the lateral recess in the triangle type stenosis was observed in the present study; however, narrowing of the lateral recess causes compression of spinal roots, and symptoms are often worsened with walking in the trefoil type due to the nature of patterned LCS (Fig. 1). Therefore, the various MRI morphology types may be a preoperative predictor of walking ability in patients with LCS. Despite these findings, further investigation must be conducted to support this observation. For instance, although it is known that LCS describes patients with symptoms related to anatomical reduction of the size of the lumbar spine [2], whether a relationship exists between the MRI morphology grade stenosis and symptoms is unclear.

This study attempted to summarize the influence of stenosis morphology on the objective assessment of the walking ability of patients with LCS. Theoretically, the degree of stenosis of the spinal cord in trefoil stenosis is more severe than that in triangle stenosis; however, this study showed that the minimum cross-sectional area in trefoil stenosis is smaller than that in triangle stenosis. Therefore, the findings of this study are consistent with the results in the literature. Although there are some limitations associated with this study, the results are helpful for judging the clinical nerve function via MR examination.
There are several principle weaknesses in this study. (1) We were unable to identify a relationship between all grades (triangle: $\mathrm{A}, \mathrm{B}$, and $\mathrm{C}$, trefoil $\mathrm{A}, \mathrm{B}$, and $\mathrm{C}$, and the pin-hole group that represented the more severe stenosis characterized by Menon et al. [7]) of stenosis and walking ability in patients with LCS because of the low number of patients in these groups. Thus, further studies are required to clarify the relationship between the grading stenosis and walking ability in patients with LCS. (2) No statistical analysis was performed to compare between symmetrical and asymmetrical stenosis in groups because of a small number of cases. (3) Age, gender, depression, and comorbidities may significantly affect walking ability; however, we were unable to assess these factors separately and independently. (4) Multiple grades of stenosis that co-existed were not evaluated in the same lumbar spine. Hence, further studies with such data are needed. (5) Finally, walking ability and social life function might be affected by multiple preoperative factors (e.g., MRI findings and confounding factors). Thus, a multiple regression analysis should be performed with a large sample size in a multicenter study with appropriate statistical power to clarify this finding.

\section{Conclusions}

The findings suggest that the type of MRI morphology stenosis is responsible for the discrepancies between the walking ability among patients with LCS.

\section{Conflict of Interest}

No potential conflict of interest relevant to this article was reported.

\section{Acknowledgments}

The authors thank the staff of the Neurosurgery Unit at Imam-Hossain Hospital, Tehran, Iran.

\section{References}

1. Lurie J, Tomkins-Lane C. Management of lumbar spinal stenosis. BMJ 2016;352:h6234.

2. Genevay S, Atlas SJ. Lumbar spinal stenosis. Best Pract Res Clin Rheumatol 2010;24:253-65.

3. Bjorn S. Lumbar spinal stenosis: striving for less in- 
vasive surgery. Eur Neurol Rev 2006;6:44-7.

4. Tudor-Locke CE, Myers AM. Challenges and opportunities for measuring physical activity in sedentary adults. Sports Med 2001;31:91-100.

5. Katz JN, Harris MB. Clinical practice: lumbar spinal stenosis. N Engl J Med 2008;358:818-25.

6. Geisser ME, Haig AJ, Tong HC, et al. Spinal canal size and clinical symptoms among persons diagnosed with lumbar spinal stenosis. Clin J Pain 2007;23:7805.

7. Menon VK, Raniga SB, Al Busaidi AQ. MRI morphology of surgically treated lumbar canal stenosis: a retrospective study. J Spinal Disord Tech 2015;28:128.

8. Lee GY, Lee JW, Choi HS, Oh KJ, Kang HS. A new grading system of lumbar central canal stenosis on MRI: an easy and reliable method. Skeletal Radiol 2011;40:1033-9.

9. Lohman CM, Tallroth K, Kettunen JA, Lindgren KA. Comparison of radiologic signs and clinical symptoms of spinal stenosis. Spine (Phila Pa 1976) 2006;31:1834-40.

10. Ogikubo O, Forsberg L, Hansson T. The relationship between the cross-sectional area of the cauda equina and the preoperative symptoms in central lumbar spinal stenosis. Spine (Phila Pa 1976) 2007;32:1423-8.

11. Schizas C, Theumann N, Burn A, et al. Qualitative grading of severity of lumbar spinal stenosis based on the morphology of the dural sac on magnetic resonance images. Spine (Phila Pa 1976) 2010;35:191924.

12. Azimi P, Shahzadi S, Montazeri A. The Japanese Orthopedic Association Back Pain Evaluation Questionnaire (JOABPEQ) for low back disorders: a validation study from Iran. J Orthop Sci 2012;17:521-5.

13. Tomkins CC, Battie MC, Rogers T, Jiang H, Petersen S. A criterion measure of walking capacity in lumbar spinal stenosis and its comparison with a treadmill protocol. Spine (Phila Pa 1976) 2009;34:2444-9.

14. Nlilley E. Epsteil: lumbar spine stenosis 265. In: Youmans JR, Winn HR, editors. Youmans neurologi- cal surgery. 5th ed. Philadelphia: Elsevier/Saunders; 2011. p.2923-34.

15. Rainville J, Childs LA, Pena EB, et al. Quantification of walking ability in subjects with neurogenic claudication from lumbar spinal stenosis: a comparative study. Spine J 2012;12:101-9.

16. Tomkins-Lane CC, Battie MC. Validity and reproducibility of self-report measures of walking capacity in lumbar spinal stenosis. Spine (Phila Pa 1976) 2010; 35:2097-102.

17. Hamanishi C, Matukura N, Fujita M, Tomihara M, Tanaka S. Cross-sectional area of the stenotic lumbar dural tube measured from the transverse views of magnetic resonance imaging. J Spinal Disord 1994;7: 388-93.

18. Azimi P, Mohammadi HR, Benzel EC, Shahzadi S, Azhari S, Montazeri A. Decision-making process in patients with lumbar spinal canal stenosis. J Neurosurg Sci 2017;61:388-94.

19. Landis JR, Koch GG. The measurement of observer agreement for categorical data. Biometrics 1977;33: 159-74.

20. Kuittinen P, Sipola P, Saari T, et al. Visually assessed severity of lumbar spinal canal stenosis is paradoxically associated with leg pain and objective walking ability. BMC Musculoskelet Disord 2014;15:348.

21. Deen HG, Zimmerman RS, Lyons MK, McPhee MC, Verheijde JL, Lemens SM. Use of the exercise treadmill to measure baseline functional status and surgical outcome in patients with severe lumbar spinal stenosis. Spine (Phila Pa 1976) 1998;23:244-8.

22. Khoo LT, Fessler RG. Microendoscopic decompressive laminotomy for the treatment of lumbar stenosis. Neurosurgery 2002;51(5 Suppl):S146-54.

23. Kleeman TJ, Hiscoe AC, Berg EE. Patient outcomes after minimally destabilizing lumbar stenosis decompression: the "Port-Hole" technique. Spine (Phila Pa 1976) 2000;25:865-70.

24. Weiner BK, Walker M, Brower RS, McCulloch JA. Microdecompression for lumbar spinal canal stenosis. Spine (Phila Pa 1976) 1999;24:2268-72. 